\title{
Birth Date and Time
}

National Cancer Institute

\section{Source}

National Cancer Institute. Birth Date and Time. NCI Thesaurus. Code C83217.

The date and time of a birth event. 